\title{
Translating evidence into healthcare policy and practice: Single versus multi-faceted implementation strategies - is there a simple answer to a complex question?
}

\author{
Gill Harvey ${ }^{1,2^{*}}$, Alison Kitson ${ }^{1,3}$
}

\begin{abstract}
How best to achieve the translation of research evidence into routine policy and practice remains an enduring challenge in health systems across the world. The complexities associated with changing behaviour at an individual, team, organizational and system level have led many academics to conclude that tailored, multifaceted strategies provide the most effective approach to knowledge translation. However, a recent overview of systematic reviews questions this position and sheds doubt as to whether multi-faceted strategies are any better than single ones. In this paper, we argue that this either-or distinction is too simplistic and fails to recognize the complexity that is inherent in knowledge translation. Drawing on organizational theory relating to boundaries and boundary management, we illustrate the need for translational strategies that take account of the type of knowledge to be implemented, the context of implementation and the people and processes involved.

Keywords: Knowledge Translation, Boundaries, Boundary Management, Evidence-Based Healthcare Copyright: $\odot 2015$ by Kerman University of Medical Sciences

Citation: Harvey G, Kitson A. Translating evidence into healthcare policy and practice: Single versus multifaceted implementation strategies - is there a simple answer to a complex question? Int J Health Policy Manag 2015; 4: 123-126. doi: 10.15171/ijhpm.2015.54
\end{abstract}

Article History:

Received: 15 February 2015

Accepted: 4 March 2015

ePublished: 5 March 2015
I $\mathrm{t}$ is generally accepted that the translation of research evidence into healthcare decision-making is both challenging and complex. Despite the language of translational pipelines and bridging the gaps between evidence and policy or practice, real-life experiences rarely follow a straightforward rational or linear pathway $(1,2)$. This is due to factors such as the contestable nature of evidence, the multiple influences on decision-making and the effect of contextual variables at a micro, meso and macro level $(3,4)$. Consequently translation strategies that attempt to recognize and manage the multiplicity of factors are proposed, often comprising a number of inter-related elements that can be tailored according to the particular setting and target audience (5). As such, common practice typically favours multifaceted approaches to translation (6). However, a recent overview of systematic reviews questions the relevance and validity of such claims. Having reviewed 25 systematic reviews of interventions to change healthcare professional behaviour, the authors concluded that they had found "no compelling evidence that multifaceted interventions are more effective than single-component interventions as commonly believed" (7) (p.21).

So what does this mean in terms of moving forward with knowledge translation and translational science in healthcare? Are we wasting limited resources on complex, over-engineered translation strategies when simpler - and cheaper - ones would do an equally good job? How do we know whether to adopt a single or multi-faceted intervention? And is the issue really as straightforward as deciding between a single and multi-faceted approach - or is the answer to this question as contingent, complex and context-dependent as the process of knowledge translation itself? These are important issues to address given universal economic constraints in healthcare and the need to ensure that the translational initiatives we establish are as cost-efficient and effective as possible.

Addressing issues of complexity and tailoring interventions to context is something that we have been studying over a number of years in the process of developing, evaluating and refining a framework to guide knowledge translation. This framework - the Promoting Action on Research Implementation in Health Services framework, or PARIHS as it is commonly known - proposes that successful translation is a function of the interplay between the research evidence (how robust it is and how it aligns with clinical, patient and local evidence), the context in which translation is happening (in terms of its receptivity to new ideas, the prevailing culture, leadership and orientation to evaluation and learning) and the ways in which the process is facilitated (how and by whom) (8-10). Central to the PARIHS framework is the idea of one or more individuals in a facilitator role using facilitation methods and processes in a flexible way to respond to the contingent nature of evidence and the variable influence of context (11-13). Thus facilitation functions as the active ingredient' in translation, assessing and diagnosing barriers and enablers in the particular setting and devising and implementing appropriate translational strategies. From the early development of PARIHS, we recognized that a whole range of approaches to facilitation could be appropriate, ranging from goal or task-focused facilitation to more holistic, emancipatory facilitation, depending on the receptivity of the 
context and the 'fit' of the evidence with local policy, practice and priorities (13). So, for example, in a situation where the context is generally receptive to new ideas (in terms of its learning culture, leadership support, staff involvement and engagement) and the research evidence aligns closely with clinical experience, patient needs and local priorities, then a relatively simple facilitation approach is required. However, in alternative settings where the environment is challenging and unreceptive to change and the evidence is disputed, more intensive facilitation is needed to identify and address the barriers that exist at a local or organizational level. In turn, this requires facilitators to have a sophisticated set of skills in negotiation, consensus development and conflict management. The common feature across this continuum of facilitation approaches is that they are fundamentally concerned with enabling others to act, as opposed to telling, coercing or persuading. However, choices still need to be made in terms of knowing how to select the most appropriate facilitation strategy for particular circumstances and with particular groups of healthcare staff, managers or policy-makers.

In thinking about how to operationalize the concept of facilitation in practice, a key part of the facilitator's role involves analyzing and managing contextual factors at a local, organizational and health system level. At a local level, this involves assessing issues related to 'readiness to change', for example, individual and collective team level motivation and capability to introduce the required changes (14). At an organizational level, factors such as senior leadership and management support, alignment with strategy and priorities and the level of absorptive capacity become important considerations (15). External influences within the wider health system context are also important, including, for example, incentives, mandates and regulatory standards that could help or hinder in terms of reinforcing the proposed changes. Whilst facilitators might not be able to directly influence the external context, they need to be aware of it and adopt a strategy of 'informed opportunism' (16) (p.10) to align the proposed change/s to external drivers where possible. Ability to work across these different contextual levels and to manage the interplay between evidence and context clearly requires individuals who take on the facilitator role to possess a level of knowledge and skills that enables them to function in a flexible and responsive way.

In developing our notion of a facilitation continuum, whereby facilitators can move between less intensive, goal-focused facilitation to more complex, emancipatory approaches $(13,17)$, we have observed interesting parallels in research from outside of healthcare. For example, Paul Carlile's work on understanding knowledge, boundaries and strategies for moving knowledge across boundaries $(18,19)$ provides some useful insights into the debate around single versus multi-faceted interventions. Carlile's research was undertaken in the commercial sector and involved an ethnographic study of how knowledge was structured in product development in the fields of sales and marketing, design engineering, manufacturing engineering and production. Adopting a situated view of knowledge in practice, Carlile's work was premised on the belief that "the characteristics of knowledge that drive innovation within a function actually hinder problem solving and knowledge creation across functions" (18) (p. 442). Thus in building domain-specific, specialist knowledge, boundaries are created which can become problematic when attempting to move knowledge between different groups or different parts of an organization.

In studying these knowledge boundaries more closely, three different types of boundary were proposed, each progressively more complex and described as syntactic, semantic and pragmatic. Syntactic boundaries represent the simplest type of boundary and exist where the people involved in giving or receiving new knowledge experience differences in language and terminology, but are able to reach a solution by finding a common syntax. This is most likely to occur when stable conditions exist. A more complex boundary - a semantic boundary - occurs when there are differences in the interpretation of the new knowledge; consequently there needs to be some effort to establish and agree a common meaning. The most complex boundary is described as a pragmatic boundary and arises when the new knowledge introduces a high degree of novelty. In such circumstances, the issues are more than differences in language or meaning; rather knowledge is seen to be "at stake" and there are costs involved in adopting the new knowledge for some or all of the parties involved. In order to agree to and adopt the new (collective) knowledge, individuals and teams may have to adjust and adapt their existing domain-specific or specialist knowledge. This is likely to lead to processes of contestation and negotiation, involving issues such as power, roles and relationships.

Building on this boundary theory, Carlile suggested that different strategies are needed to address syntactic, semantic and pragmatic boundaries (19). At a syntactic boundary, knowledge transfer strategies, focused on information processing and built around an agreed language and set of messages, are seen to be sufficient. However, at a semantic boundary, translational strategies that aim to achieve a sense of collective meaning are required in order to address interpretive differences, for example, by engaging in shared discussion and activity. And at the most complex, pragmatic boundary, the focus needs to shift to transformational approaches encompassing processes to negotiate the knowledge differences that exist and related issues of power, vested interests and politics.

Returning to the starting point for our discussion on single versus multi-faceted interventions for knowledge translation - what is the answer? Not surprisingly, our response would be 'it depends'. When trying to change behaviour, whether at a patient, population, clinical, managerial or policy level, we are undoubtedly dealing with issues of complexity. As such, there is unlikely to be a simple answer and it would be a backwards step to advocate abandoning tailored, multifaceted interventions in favour of cheaper and simpler alternatives. Rather, we need to build upon what we have learnt so far in the field of implementation and translational science about the complex inter-relationships between what we are trying to change, the people involved in the translation process and the contextual setting in which they work. Considerable progress has been made in terms of understanding the factors that influence and impact the 
uptake of evidence into policy and practice, including the contribution that existing behavioural and organizational theories can make (20). As previous contributors to this journal have highlighted, this includes drawing on theories that have originated outside of healthcare $(21,22)$.

Most importantly, we need to apply this growing theoretical and empirical knowledge in a deliberate way when we plan, implement and evaluate translational strategies - assessing, for example, how much novelty the proposed change introduces, how people are likely to respond to it and what sort of contextual influences exist at a local, organizational and system level. Depending on the answers to questions such as these, we can then make informed decisions as to whether we need a 'transfer-translate-transformation' solution. If we are confident that the boundaries are relatively simple and straightforward, then a single implementation strategy (for example, audit and feedback) may well be appropriate. Indeed, it would be an inefficient use of resources to apply an 'all-singing-all-dancing' multi-faceted approach in such a circumstance. However, if the boundaries are more complex, so too more complex implementation strategies encompassing interactive and/or negotiated elements are called for. And as we move to these more complex translational and transformational approaches, we are likely to need a combination of different strategies, active facilitation of the process and conscious application of one or more theories and frameworks that have been developed to inform and guide implementation [see, for example, (23-25)].

The review by Squires and colleagues (7) has raised some timely and important questions. We cannot and should not waste time and resources on implementation strategies that are more complicated than they need to be. But equally we have to recognize that we are dealing with issues of complexity and that there is no 'one-size-fits-all' solution to the challenge of knowledge translation in healthcare. Many healthcare organizations that are attempting to tackle issues of translation are large and complex and multiple professional and managerial boundaries are likely to exist. Indeed, recent research into healthcare networks that have been purposefully established to accelerate translation demonstrates the effect of existing (and in some cases newly created) professional, epistemic and project team boundaries on impeding knowledge flow $(26,27)$.

A final point for consideration relates to the methods that are employed to study the processes and outcomes of knowledge translation. The research reported by Squires and colleagues (7) was an overview of systematic reviews, which employ a particular methodology to identify and appraise intervention studies and adopt a 'gold-standard' definition of evidence derived from randomized controlled trials. This opens up the debate about which methodologies are most appropriate to capture the complexities associated with knowledge translation and the recognized contingencies related to context and implementation processes. Carlile, for example, elected to study translation using an ethnographic approach. Others, such as Rycroft-Malone and colleagues (28) and Greenhalgh and colleagues (29), have adopted a realist evaluation approach in an attempt to build explanatory theory about what works, for whom, in what circumstances, how and why. Rather than seeking causal relationships between intervention and effect, the realist methodology seeks to build a causal explanation of the relationship between context, mechanisms (the response that the intervention or components of the intervention generates) and outcomes (30). Just as we are advocating a need to be flexible and responsive in designing implementation strategies, so too we would suggest that we need to acknowledge issues of complexity in designing evaluation studies and recognize the need for a range of research methodologies to adequately capture the contingent and context-dependent nature of translational processes and outcomes.

\section{Ethical issues}

Not applicable.

Competing interests

Authors declare that they have no competing interests.

\section{Authors' contributions}

$\mathrm{GH}$ and AK conceptualized the content of the paper; GH prepared and edited drafts; $\mathrm{GH}$ and $\mathrm{AK}$ agreed the final version.

\section{Authors' affiliations}

${ }^{1}$ School of Nursing, University of Adelaide, Adelaide, Australia. ${ }^{2}$ Manchester Business School, University of Manchester, Manchester, UK. ${ }^{3}$ Central Adelaide Local Health Network (CALHN), Adelaide, Australia.

\section{References}

1. Greenhalgh T, Wieringa $S$. Is it time to drop the "knowledge translation' metaphor? A critical literature review. J R Soc Med 2011; 104: 501-9. doi: 10.1258/jrsm.2011.110285

2. Davies H, Nutley S, Walter I. Why 'knowledge transfer' is misconceived for applied social research. J Health Serv Res Policy 2008; 13: 188-90. doi: 10.1258/jhsrp.2008.008055

3. Dopson S, Fitzgerald L. Knowledge to Action? Evidence-Based Health Care in Action. New York: Oxford University Press; 2005.

4. Ferlie E, Dopson S, Fitzgerald L, Locock L. Renewing policy to support evidence-based health care. Public Administration 2009; 87: 837-52. doi: 10.1111/j.1467-9299.2009.01796.x

5. Wensing M, Oxman A, Baker R, Godycki-Cwirko M, Flottorp S, Szecsenyi J, et al. Tailored implementation for chronic diseases (TICD): A project protocol. Implement Sci 2011; 6: 103.

6. Bero LA, Grilli R, Grimshaw JM, Harvey E, Oxman AD, Thomson MA. Closing the gap between research and practice: an overview of systematic reviews of interventions to promote the implementation of research findings. The Cochrane Effective Practice and Organization of Care Review Group. British Medical Journal 1998; 317: 465-8.

7. Squires J, Sullivan K, Eccles M, Worswick J, Grimshaw J. Are multifaceted interventions more effective than single-component interventions in changing health-care professionals' behaviours? An overview of systematic reviews. Implement Sci 2014; 9: 152.

8. Kitson A, Harvey G, McCormack B. Enabling the implementation of evidence based practice: a conceptual framework. Quality Health Care 1998; 7: 149-59.

9. Rycroft-Malone J, Kitson A, Harvey G, McCormack B, Seers K, Titchen $A$, et al. Ingredients for change: revisiting a conceptual framework. Qual Saf Health Care 2002; 11: 174-80.

10. Kitson A, Rycroft-Malone J, Harvey G, McCormack B, Seers K, Titchen A. Evaluating the successful implementation of evidence into practice using the PARIHS framework: theoretical and practical challenges. Implement Sci 2008; 3: 1.

11. Rycroft-Malone J, Seers K, Titchen A, Harvey G, Kitson A, McCormack B. What counts as evidence in evidence-based 
practice? J Adv Nurs 2004; 47: 81-90.

12. McCormack B, Kitson A, Harvey G, Rycroft-Malone J, Titchen A, Seers K. Getting evidence into practice: the meaning of 'context'. J Adv Nurs 2002; 38: 94-104.

13. Harvey G, Loftus-Hills A, Rycroft-Malone J, Titchen A, Kitson A, McCormack B, et al. Getting evidence into practice: the role and function of facilitation. J Adv Nurs 2002; 37: 577-88.

14. Weiner B. A theory of organizational readiness for change. Implement Sci 2009; 4: 67.

15. Harvey G, Jas P, Walshe K. Analysing organisational context: case studies on the contribution of absorptive capacity theory to understanding inter-organisational variation in performance improvement. BMJ Qual Saf 2014. doi: 10.1136/ bmjqs-2014-002928

16. Bate P. Context is everything. Perspectives on Context: $A$ selection of essays considering the role of context in successful quality improvement. London: The Health Foundation; 2014.

17. Seers K, Cox K, Crichton N, Edwards R, Eldh A, Estabrooks C, et al. FIRE (facilitating implementation of research evidence): a study protocol. Implement Sci 2012; 7: 25.

18. Carlile PR. A Pragmatic View of Knowledge and Boundaries: Boundary Objects in New Product Development. Organ Sci 2002; 13: 442-55. doi: 10.1287/orsc. 13.4.442.2953

19. Carlile PR. Transferring, Translating, and transforming: an integrative framework for managing knowledge across boundaries. Organ Sci 2004; 15: 555-68. doi: 10.1287/ orsc.1040.0094

20. Grol R, Bosch M, Hulscher M, Eccles M, Wensing M. Planning and studying improvement in patient care: the use of theoretical perspectives. Milbank Q 2007; 85: 93-138.

21. Burton CR, Rycroft-Malone J. Resource based view of the firm as a theoretical lens on the organisational consequences of quality improvement. Int J Health Policy Manag 2014; 3: 113-5. doi: $10.15171 /$ ijhpm.2014.74

22. Ferlie E. Resource based view: a promising new theory for healthcare organizations: Comment on "Resource based view of the firm as a theoretical lens on the organisational consequences of quality improvement". Int J Health Policy Manag 2014; 3: 3478. doi: 10.15171/ijhpm.2014.107

23. Michie S, van Stralen M, West R. The behaviour change wheel: A new method for characterising and designing behaviour change interventions. Implement Sci 2011; 6: 42.

24. Tabak RG, Khoong EC, Chambers DA, Brownson RC. Bridging Research and Practice. American Journal of Preventive Medicine 2012; 43: 337-50. doi: 10.1016/j.amepre.2012.05.024

25. Rycroft-Malone J, Bucknall T. Models and frameworks for implementing evidence-based practice: linking evidence to action. Chichester, England: Wiley-Blackwell; 2010.

26. Kislov R. Boundary discontinuity in a constellation of interconnected practices. Public Adm 2014; 92: 307-23. doi: 10.1111/padm. 12065

27. Currie G, El Enany N, Lockett A. Intra-professional dynamics in translational health research: The perspective of social scientists. Soc Sci Med 2014; 114: 81-8. doi: 10.1016/j. socscimed.2014.05.045

28. Rycroft-Malone J, Wilkinson J, Burton CR, Harvey G, McCormack B, Graham I, et al. Collaborative action around implementation in Collaborations for Leadership in Applied Health Research and Care: towards a programme theory. J Health Serv Res Policy 2013; 18: 13-26. doi: 10.1177/1355819613498859

29. Greenhalgh T, Humphrey C, Hughes J, Macfarlane F, Butler C, Pawson R. How do you modernize a health service? A realist evaluation of whole-scale transformation in london. Milbank $Q$ 2009; 87: 391-416. doi: 10.1111/j.1468-0009.2009.00562.x

30. Pawson R. The Science of Evaluation: A Realist Manifesto. London: Sage; 2013. 